\title{
In vivo dendrite regeneration after injury is different from dendrite development
}

\author{
Katherine L Thompson-Peer, Laura DeVault, Tun Li, Lily Yeh Jan, and Yuh Nung Jan \\ Howard Hughes Medical Institute, Department of Physiology, Department of Biochemistry and Biophysics, \\ University of California at San Francisco, San Francisco, California 94158, USA
}

\begin{abstract}
Neurons receive information along dendrites and send signals along axons to synaptic contacts. The factors that control axon regeneration have been examined in many systems, but dendrite regeneration has been largely unexplored. Here we report that, in intact Drosophila larvae, a discrete injury that removes all dendrites induces robust dendritic growth that recreates many features of uninjured dendrites, including the number of dendrite branches that regenerate and responsiveness to sensory stimuli. However, the growth and patterning of injury-induced dendrites is significantly different from uninjured dendrites. We found that regenerated arbors cover much less territory than uninjured neurons, fail to avoid crossing over other branches from the same neuron, respond less strongly to mechanical stimuli, and are pruned precociously. Finally, silencing the electrical activity of the neurons specifically blocks injury-induced, but not developmental, dendrite growth. By elucidating the essential features of dendrites grown in response to acute injury, our work builds a framework for exploring dendrite regeneration in physiological and pathological conditions.
\end{abstract}

[Keywords: dendrite regeneration; dendrite regrowth; neuronal injury; Drosophila larvae; dendrite patterning; sensory neurons]

Supplemental material is available for this article.

Received April 18, 2016; revised version accepted July 25, 2016.

Dendrites can be damaged by a number of insults. After a stroke, in the region adjacent to the site of ischemia known as the penumbra or peri-infarct, neurons exhibit abnormalities in dendrite shape and architecture such as blebbing and beading (Hori and Carpenter 1994; Brown et al. 2008; Li and Murphy 2008; Murphy and Corbett 2009). Traumatic brain injury, often observed in athletes and soldiers, also can cause dendritic damage (Gao et al. 2011; Xiong et al. 2013). Perinatal hypoxia causes simplification of Purkinje neuron dendrites in mice (Zonouzi et al. 2015). Defects in dendrite morphology and electrophysiology are observed early in mouse models of Huntington's disease, after behavioral symptom onset but before neurodegenerative cell death begins (Klapstein et al. 2001).

While dendrite injury is clearly clinically relevant, almost nothing is known about whether and under what conditions dendrites might be able to recover after injury. One of the only studies on dendrite regeneration comes from Ramon y Cajal (1928), who observed that there were different potential responses to dendrite injury. Ramon y Cajal (1928) observed no dendrite regrowth or the formation of retraction bulbs following a slicing injury across the dendrite arbors of pyramidal neurons in the ce-

Corresponding author: yuhnung.jan@ucsf.edu Article is online at http://www.genesdev.org/cgi/doi/10.1101/gad.282848. 116. rebral cortex or the spinal cord gray matter. However, the growth of new dendrites was evident after contusion injury to the spinal cord. These observations indicate that, depending on the neuron type and form of injury, some neurons are capable of regenerating their dendrites while others cannot, likely due to a combination of cell-intrinsic and environmental factors. While cultured neuron assays in which axons and dendrites regrow after dissociation have advanced our understanding of many topics of neuronal cell biology (Barnes and Polleux 2009), they lack the environmental context of in vivo observations.

Dendrite arborization (da) neurons are sensory neurons in the Drosophila larval peripheral nervous system (PNS) that can be grouped into four classes based on the classspecific expression of genes and the complexity of their dendrite arbors (Jan and Jan 2010). Class I da neurons have the simplest dendritic arbor, while class IV da neurons have the most complex arbor (Grueber et al. 2003a). The development of these arbors is highly stereotyped by class and neuronal identity. Class I neurons form new branches only during embryogenesis and early larval development, while class IV da neurons continuously form

(C) 2016 Thompson-Peer et al. This article is distributed exclusively by Cold Spring Harbor Laboratory Press for the first six months after the full-issue publication date (see http://genesdev.cshlp.org/site/misc/ terms.xhtml). After six months, it is available under a Creative Commons License (Attribution-NonCommercial 4.0 International), as described at http://creativecommons.org/licenses/by-nc/4.0/. 
new branches throughout larval development as they scale with the growth of the animal. Notably, class III and class IV dendrites tile the entire body wall, with each neuron responsible for covering one section of a hemisegment without crossing over the dendrites from adjacent neurons (Grueber et al. 2002). Within that territory, dendrites avoid crossing over other branches from the same neuron (Grueber et al. 2003b). Later, during the pupal stage, some of these arbors are pruned to make way for the growth of adult dendrites, while other neurons die (Kuo et al. 2005; Williams and Truman 2005).

Previous studies have shown that at least some of these da neurons are capable of regrowing dendrites after injury, and one has shown that the canonical axon injury-sensing pathway DLK/Wallenda is dispensable for dendrite regeneration (Sugimura et al. 2003; Song et al. 2012; Stone et al. 2014). However, little was known about the properties and the quality of the regenerated dendrites. Here, in order to understand how neurons recover from dendrite injury, we used the wealth of knowledge about normal dendrite patterning of da neurons to explore fundamental features of dendrite regeneration and define the ways in which regenerated dendrites are similar to and different from dendrites of uninjured neurons.

\section{Results}

Sensory neurons regenerate branch number but not architecture after injury

To study dendrite regeneration after dendrite injury, we selectively remove all of the dendrite branches of da neurons in Drosophila larvae using a two-photon laser. This "balding" injury does not kill the neuron but instead allows us to examine the response of a neuron to an extreme insult in intact animals, as shown previously (Stone et al. 2014). We balded the neuron, imaged it $24 \mathrm{~h}$ later when neurons have begun to re-establish some dendrites to confirm the injury (Supplemental Fig. S1A), and then imaged it again $2 \mathrm{~d}$ later (72 $\mathrm{h}$ after injury) to assess the extent of regeneration (Fig. 1A). By $72 \mathrm{~h}$ after balding, neurons had regenerated many dendrite branches (Supplemental Fig. S1A). We compared these balded neurons with mock-injured control neurons, which are uninjured neurons within the same larvae. These control neurons are therefore perfectly matched for developmental stage and growth conditions and have gone through the same process of repeated mounting for microscopy. To quantify the extent of growth after balding or mock injury, we counted the number of branches, measured the total length of the dendrite arbor, and determined the size of the territory covered by the dendrite arbor. This study thus goes beyond what had been reported previously, which counted only the fraction of neurons that exhibited any dendrite growth after balding without either quantification of the amount of growth after injury or comparison with uninjured neurons (Song et al. 2012; Stone et al. 2014).

We began by examining the regeneration of the ddaC subtype of class IV da neurons. In order to eliminate competition with adjacent uninjured neurons, we ablated the neurons to the anterior and posterior of the balded neuron of interest. We found that, in the absence of invasive growth from adjacent uninjured neurons, the class IV da neurons balded at $48 \mathrm{~h}$ AEL (after egg laying) regenerated the correct number of dendrite branches to match an uninjured neuron but covered only approximately half of the territory that an uninjured neuron covers (Fig. 1B-E). Sholl analysis showed that these regenerated arbors are more densely branched close to the cell body (Fig. 1F). Thus, while balded neurons can regenerate the correct number of dendrite branches, the morphology of this new arbor is drastically different from that of an uninjured arbor, covering only half the territory.

Class I da neurons extend a simple arbor early in development and do not continue to form new branches during the later larval stages. As the larva gets bigger, the scaling growth of class IV da neurons includes both forming new branches and lengthening of existing branches, while class I da neurons only lengthen existing branches. In agreement with a previous study (Stone et al. 2014), we found that the normally stable ddaE class I da neurons regrow new branches to match the number of branches of an uninjured neuron (Fig. 2A). However, we found that regenerated arbors have a very different architecture, including a much shorter total branch length and altered organization, as compared with uninjured dendrites. Thus, regeneration of the correct number of branches, but not the morphology of an uninjured arbor, is characteristic of both class IV and class I da neurons.

To see whether the developmental stage altered the architecture of the regenerated class IV dendrites, we balded $\mathrm{ddaC}$ neurons at earlier stages of larval development without ablating the adjacent uninjured neurons. At all stages examined, including just after hatching (28 h AEL), later first instar (36 h AEL), and second instar (48 h AEL), all class IV da neurons were capable of regenerating their dendrites (Supplemental Fig. S1H-L). These younger neurons formed enough new branches to reach the same number of branches as an uninjured neuron (Supplemental Fig. S1I). We observed significant invasion by the adjacent uninjured neurons into the balded neurons' territory, similar to the invasion by adjacent uninjured neurons into the newly unoccupied territory of a neuron that had been recently ablated (Parrish et al. 2009).

To test whether older neurons lose regenerative competence, we balded class IV neurons at $72 \mathrm{~h} \mathrm{AEL}$, when invasion by adjacent uninjured neurons growing into newly vacated territory is minimal (Parrish et al. 2009). After balding, neurons still exhibited robust regrowth of their dendrites (Supplemental Fig. S1B,C,F), although not enough to catch up to uninjured neurons. Notably, the branch number and total length increased to the same degree with or without injury (Supplemental Fig. S1D,E). Regenerated arbors were still smaller in total size and more dense near the cell body than uninjured arbors (Supplemental Fig. S1G).

A subset of class I and class IV da neurons persists after metamorphosis, including the class I neuron ddaE and the class IV neuron ddaC; they undergo developmental pruning and regenerate their arbors for the adult animal 
Thompson-Peer et al.
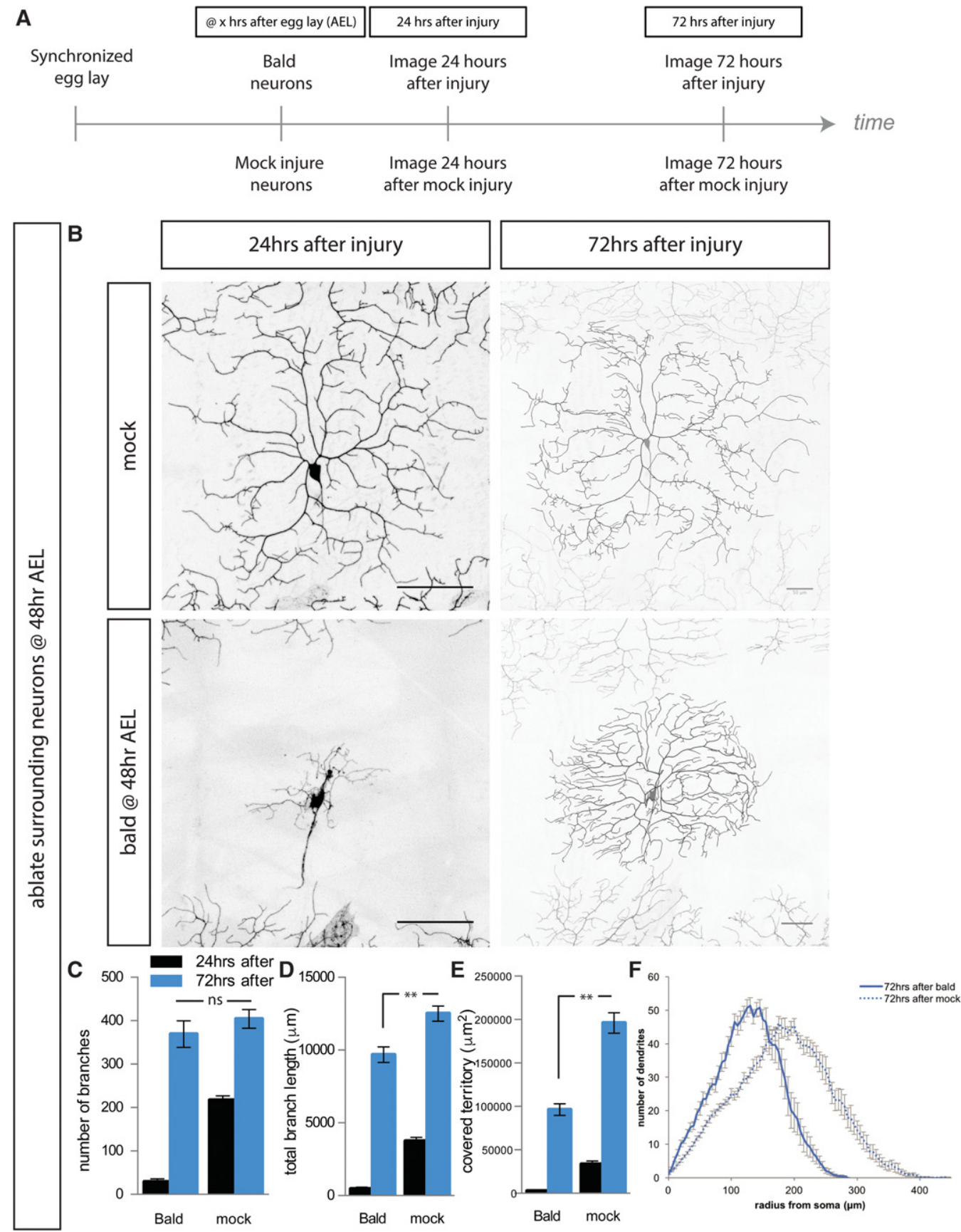

Figure 1. Class IV da neurons regenerate branch number, but not territory coverage, after all dendrites are severed at $48 \mathrm{~h}$ after egg laying (AEL). (A) Schema of experiments in this study. After synchronized egg laying, neurons were either balded or uninjured, usually at 48 or 72 $\mathrm{h}$ AEL. Neurons were mounted and imaged $\sim 24 \mathrm{~h}$ after injury or mock injury to confirm that all dendrites had been severed and again $72 \mathrm{~h}$ after injury or mock injury to assess regeneration. $(B)$ Representative images of ddaC neurons when adjacent anterior and posterior neurons have been ablated $24 \mathrm{~h}$ (left) and $72 \mathrm{~h}$ (right) after either balding (bottom) or mock injury (top). (C,D) By $72 \mathrm{~h}$ after balding (blue), neurons regenerate the number of branches and almost regenerate the arbor length of an uninjured neuron. $(E)$ Neurons recover only about half of the body wall territory of an uninjured neuron. $(F)$ Sholl analysis of dendrite complexity $72 \mathrm{~h}$ after injury for balded (solid) and control (dashed) neurons. ( $\left.^{* *}\right) P<0.0001$; (ns) $P>0.05$, by one-way ANOVA with Tukey's multiple comparisons test. Bar, $50 \mu \mathrm{m}$. $n=16$ balded neurons and 15 mock neurons.

(Williams and Truman 2005; Shimono et al. 2009). To test whether only neurons destined to persist after metamorphosis would be able to regenerate dendrites after injury during the larval phase, we balded neurons that die during metamorphosis instead of regenerating, specifically the class III da neuron ddaA and the class IV da neuron vdaB (Williams and Truman 2005). We found that, similar to the class I neuron ddaE and class IV neuron ddaC, which 
A
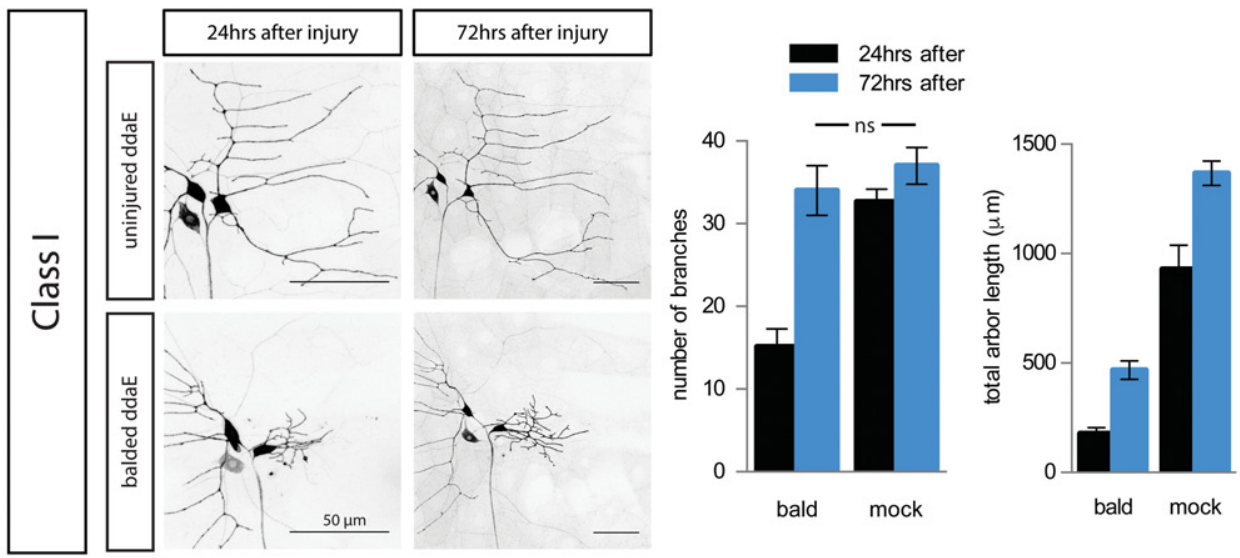

B
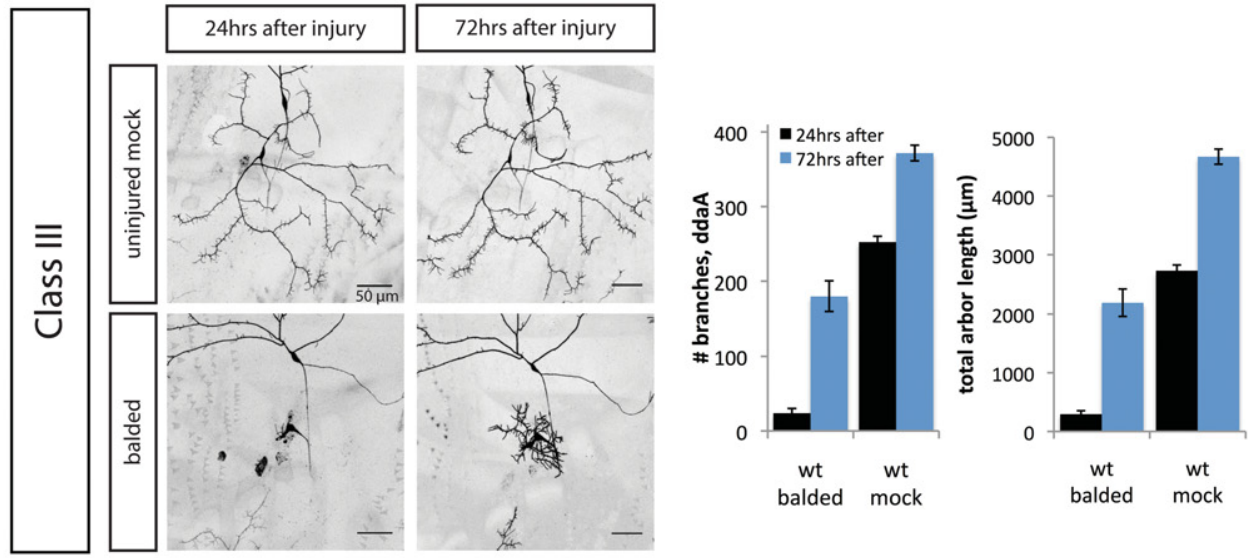

C

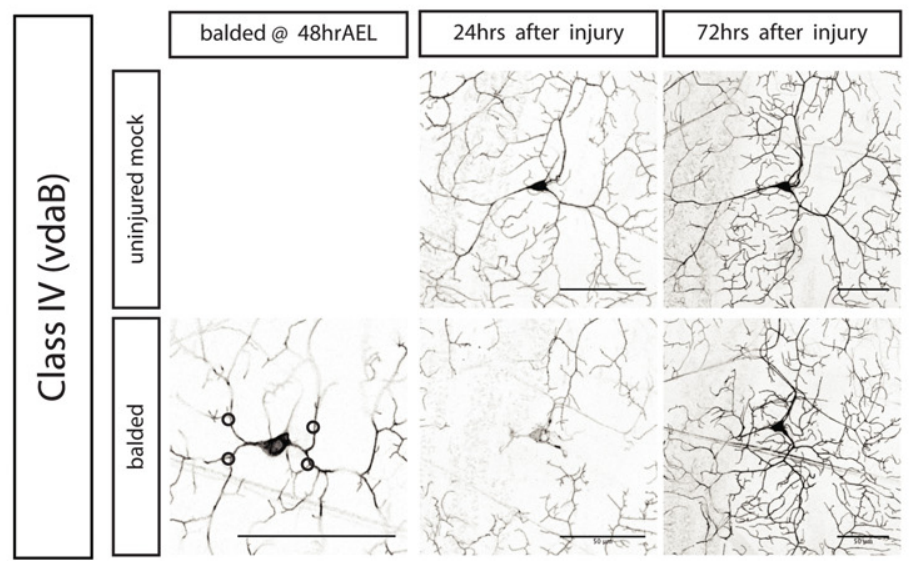

Figure 2. Other da neurons, including class I, class III, and other class IV neurons, can regrow dendrites after injury. (A) The class I da neuron ddaE, which does not extend new branches in the absence of injury, forms new branches after balding injury to recover the number of branches, but not the total arbor length, of an uninjured neuron. (ns) $P>0.05$, by one-way ANOVA with Tukey's multiple comparisons test. $n=9$ balded neurons and 11 mock neurons. $(B)$ The class $\mathrm{III}$ da neuron ddaA extends new terminal branch spikes after balding at $72 \mathrm{~h}$ AEL and recovers some of the dendrite length of an uninjured neuron. $n=10$ balded neurons and 16 mock neurons. $(C)$ The class IV da neuron $\mathrm{vdaB}$, which dies during metamorphosis, extends new dendrite branches after balding. $n=3$ balded neurons and 15 mock neurons. Bar, $50 \mu \mathrm{m}$.

undergo pruning and regrowth in the pupae, all of the class III ddaA neurons and the class IV vdaB neurons that die during the pupal phase were able to regrow new dendrites in the larvae after balding (Fig. 2B,C).
Using class I da neurons, we studied the relationship between developmental branching and after-injury dendrite branching. We found that RNAi-mediated knockdown of $A k t$, which causes underbranching phenotypes 
in uninjured neurons, also resulted in less regenerative branching after a dendrite branch was cut off (Supplemental Fig. S2A). Similarly, manipulations that cause overbranching phenotypes in uninjured neurons, such as the ectopic expression of the transcription factors cut or knot/collier, also resulted in overbranching after injury (Supplemental Fig. S2B; Grueber et al. 2003a; Kim et al. 2006; Hattori et al. 2007; Jinushi-Nakao et al. 2007; Crozatier and Vincent 2008). Thus, the mechanisms that determine branch number during early development also determine branch number after injury.

\section{Regenerated dendrites violate self-avoidance}

The dendrite arbors of class III and class IV da neurons cover the body wall of the larvae. An essential feature that allows them to cover the body wall is that these dendrites self-avoid, meaning that dendrite branches do not touch or cross over other branches of the same neuron (Grueber et al. 2003b). Self-avoidance and tiling allow for nonredundant detection of sensory stimuli by dendrite arbors that do not overlap. Compared with uninjured neurons, we found that regenerated arbors show a dramatic increase in the number of dendrites crossing over sister branches of the same arbor (Fig. 3A, quantified in E).

Fine $\mathrm{Z}$ optical sectioning can distinguish between dendrite self-crossing events where branches are in contact with one another (contacting crossings) and self-crossing events where branches are not in contact with one another when one branch has grown above or below the two-dimensional (2D) layer where dendrites normally exist between the extracellular matrix (ECM) and epidermal cells (noncontacting crossings) (Supplemental Fig. S3A; Han et al. 2012). These two types of morphological patterning defects are normally prevented by two distinct mechanisms. Noncontacting crossings are prevented by integrin-mediated adhesion to the ECM, which restricts dendrites to the 2D plane between the ECM and the epidermal cells (Han et al. 2012; Kim et al. 2012). Contacting crossings within that $2 \mathrm{D}$ plane are prevented by contactmediated repulsion by the transmembrane protein DSCAM (Hughes et al. 2007; Matthews et al. 2007; Soba et al. 2007). In neurons that had been balded at $72 \mathrm{~h}$ AEL, we found an increase in both contacting and noncontacting self-crossings as compared with uninjured neurons (Fig. 3A, quantified in E).

To determine the mechanism that allows regenerating dendrites to ignore the patterning cues that confer selfavoidance, we tested three approaches to restore selfavoidance to regenerated dendrites. First, we reasoned that self-avoidance pathways present in younger neurons could have been shut off in the older neurons reforming arbors after balding. To address this, we balded younger neurons at $48 \mathrm{~h}$ AEL but found that the self-avoidance defects were worse in the regenerated arbors of younger neurons (Fig. 3B). This suggested that the self-crossing defects of regenerating dendrites are not the result of developmental shutoff in older neurons.

In a second approach, we overexpressed integrins to suppress noncontacting crossings. Integrins located on the dendrite plasma membrane mediate adhesion to the ECM. The Drosophila genome has five integrin a subunits (encoded by mew, inflated, scab, aPS4, and aPS5) and two integrin $\beta$ subunits (encoded by mys and $\beta$ int- $v$ ) (Brower 2003). As seen previously, we observed that overexpressing the integrin $\alpha$ and $\beta$ subunits mys and mew eliminates the rare noncontacting crossings of uninjured neurons (Fig. 3C, quantified in E, "mock"; Han et al. 2012). We tested whether this integrin overexpression could rescue the noncontacting self-crossings observed in regenerated neuron arbors but found that, instead of rescuing self-crossings, the absolute number of self-crossing events was increased by integrin overexpression. Rather, the balance of self-crossing events in regenerated arbors was shifted from being evenly split between contacting and noncontacting crossings in wild type to mostly contacting crossings when integrins were overexpressed (Fig. 3C, quantified in E). In the presence of integrin overexpression, regenerated dendrites sometimes even fasciculated together into dendrite bundles, adhering strongly to the ECM and failing to respect contact-mediated repulsion pathways. Dendrite fascicles were never observed in uninjured neurons. Thus, while not able to eliminate crossings, integrin overexpression transformed many self-crossings into contacting self-crossings, resulting in decreased coverage of the body wall territory (Supplemental Fig. S3C).

In a third approach, we overexpressed DSCAM to suppress contacting crossings. The transmembrane protein DSCAM, which exhibits extensive alternative splicing, prevents contacting crossings (Hughes et al. 2007; Matthews et al. 2007; Soba et al. 2007). As there are very few contacting crossings in wild-type neurons to begin with, overexpressing a DSCAM splice isoform has little effect on the self-crossings of uninjured neurons (Fig. 3D, quantified in E). In regenerated arbors, almost all contacting crossings are eliminated when DSCAM is overexpressed, shifting the balance to all noncontacting self-crossings (Fig. 3D, quantified in E) without reducing the absolute number of self-crossing events. Thus, both integrin overexpression and DSCAM overexpression fail to rescue the total number of self-crossing events and instead antagonistically shift the balance of self-crossing events between contacting and noncontacting crossings. Altogether, this suggests that regenerating dendrites override multiple kinds of patterning cues to violate self-avoidance during regrowth, resulting in an arbor with a different architecture than an uninjured neuron.

We wondered how well the self-avoidance transmembrane proteins might be trafficked in regenerated dendrites. We looked at the localization of the GFP-tagged DSCAM transgene, which eliminates contacting crossings in regenerated neurons (Fig. 3D, quantified in E). In uninjured neurons, DSCAM-GFP is present in punctate accumulations in both the primary and terminal branches of class IV da neurons (Supplemental Fig. S3D, top row; Soba et al. 2007). In regenerated arbors, DSCAM-GFP is present in many but not all primary and terminal branches as well (Supplemental Fig. S3D, middle and bottom rows). 


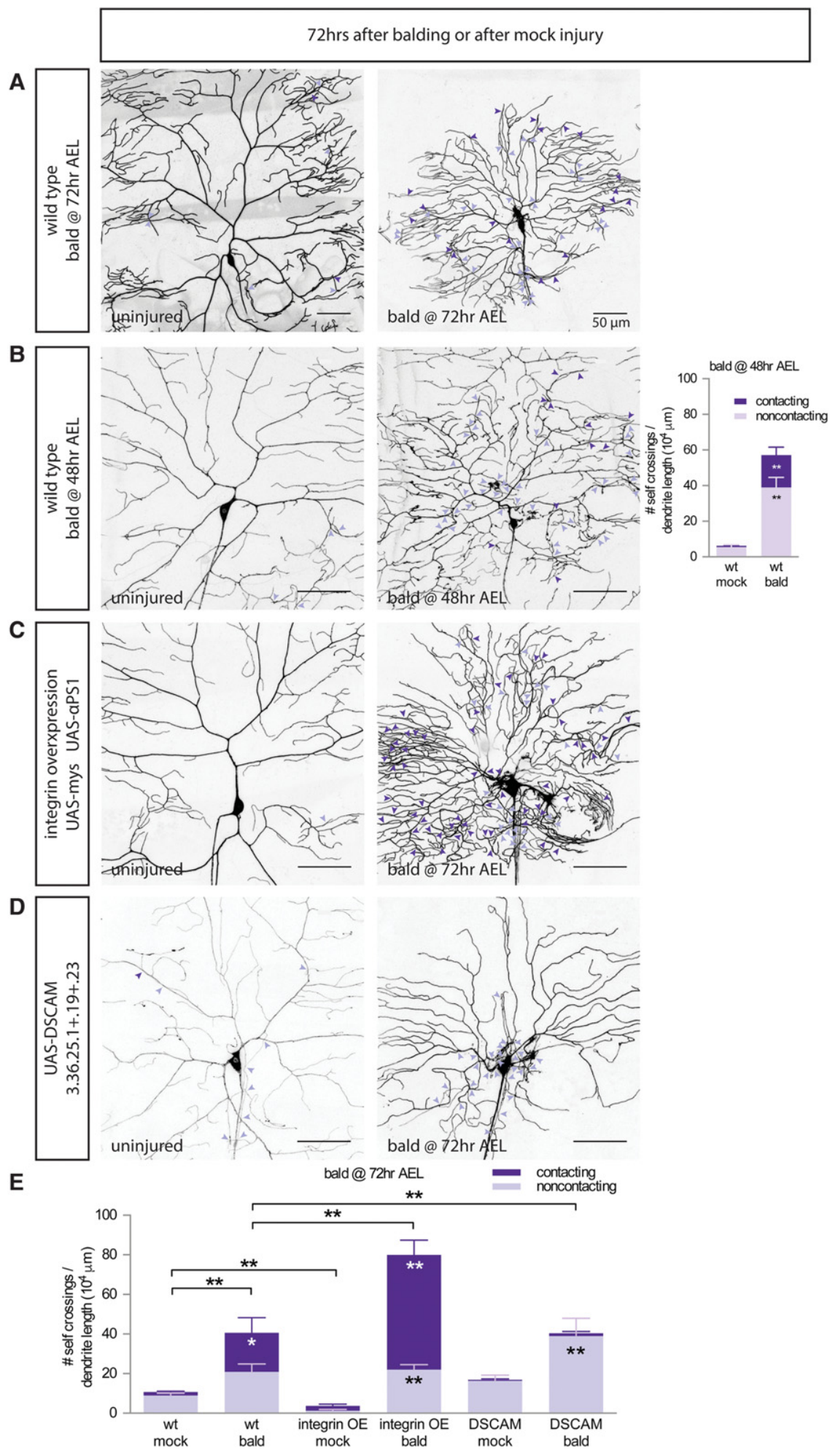

Figure 3. Regenerated dendrites have defects in both integrin-mediated and DSCAM-mediated self-avoidance. $(A, B)$ Wild-type neurons balded at $72 \mathrm{~h}$ AEL $(A)$ or $48 \mathrm{~h}$ AEL $(B)$ regenerate dendrites with significantly more contacting (dark-purple arrowheads) and noncontacting (light-purple arrowheads) self-crossings than uninjured arbors. $n=10$ mock wild-type neurons and 9 balded wild-type neurons at $48 \mathrm{~h}$ AEL. (C) Overexpressing the integrin subunits mys and $\alpha$-PS1 rescues noncontacting self-crossings in uninjured neurons but, in regenerated dendrites only, increases the number of contacting self-crossings. (D) Overexpressing a DSCAM splice isoform in regenerating neurons increases the fraction of noncontacting crossings but does not rescue the total number of selfcrossings. (E) Quantification of contacting (dark purple) and noncontacting (light purple) self-crossings in wild-type, integrinoverexpressing, and DSCAM-overexpressing neurons uninjured or balded at $72 \mathrm{~h}$ AEL. $n=6$ wild-type mock neurons, 6 wild-type balded neurons, 8 integrin mock neurons, 9 integrin balded neurons, 5 DSCAM mock neurons, and 4 DSCAM balded neurons. For both graphs, comparisons between mock versus bald neurons of the same genotype of the same type of self-crossing (contacting in dark purple or noncontacting crossings in light purple) are labeled within the bars by one-way ANOVA with Sidak's multiple comparisons test. $\left(^{*}\right) P<0.05$; $\left(^{* *}\right) P<0.01$. Comparisons of the fraction of all crossings that are contacting crossings are labeled above the bars by one-way ANOVA with Sidak's multiple comparisons test. $\left({ }^{* *}\right) P<0.01$. Bar, $50 \mu \mathrm{m}$.
Time-lapse imaging showed that dendrite tips encountering other branches of the same dendrite arbor are likely to turn or retract (Han et al. 2012). Given the defect in self-avoidance, we asked whether regenerated dendrite tips exhibit normal extension/retraction dynamics when they encounter another branch of their same arbor. We found that regenerated dendrites have more total encounters with other branches of the same arbor over a 30-min imaging period and that a greater fraction of those branch tips will fail to turn away or retract after the encounter (Supplemental Fig. 3E). 


\section{Regenerated dendrites restore partial function}

In order to test whether regenerated dendrites are functional, we filleted Drosophila larvae and recorded action potentials from class III neurons in response to a gentle touch stimulus. Class III da neurons have been shown to fire a burst of action potentials in response to a poke from a probe stimulus, with increasing displacements resulting in more spikes (Yan et al. 2013). We compared class III ddaA neurons that had been recently balded $(\sim 5$ $\mathrm{h}$ prior to recording) with control uninjured neurons and neurons that had been allowed $3 \mathrm{~d}$ to regenerate after balding. We applied the probe stimulus to one of two locations on the fillet: near the cell body where the dendrite arbor is densest and far from the cell body at the hemisegment boundary where regenerated dendrites often fail to reach. We found that recently balded neurons, which have no dendrites, have no response to mechanical stimulation of any strength applied at either location (Fig. 4A,B, gray markers). In contrast, both control neurons and neurons with regenerated dendrites responded to strong stimuli applied at either location, demonstrating that the regenerated dendrites are, in fact, functional (Fig. 4A-D).

Regenerated arbors do not recover full function. The number of action potentials elicited by mechanical stimulation is significantly reduced in regenerated neurons compared with uninjured control neurons $(P<0.05$, twoway ANOVA) (Fig. 4A,B). This demonstrates that regenerated class III dendrite arbors detect sensory stimuli differently from uninjured neurons, potentially due to the alterations that we observed in dendrite architecture (Fig. 2B).

\section{Regenerated dendrites are deficient in pruning}

After initial outgrowth, dendrite pruning is a subsequent step in the refinement of neuronal connectivity in many organisms (Schuldiner and Yaron 2015). In Drosophila, class IV da neurons undergo developmental pruning during metamorphosis as pupae (Kuo et al. 2005; Williams and Truman 2005). Calcium transients occur in specific branch units followed by dendrite branch severing proximal to the cell body, fragmentation of the distal dendrites, and engulfment by the adjacent epidermal cells (Kanamori et al. 2013; Han et al. 2014). Following complete clearance of the dendrite arbor, a new dendrite arbor is elaborated for the adult animal. Would the alterations in dendritic architecture that we observed due to injury have any effects on later dendrite refinement, pruning, and regrowth in the adult?

In order to look at the consequences of the architectural alterations that we observed in regenerated class IV arbors, we examined pruning during the pupal phase in neurons that had gone through balding and regeneration as larvae. We found that all of the regenerated arbors that we observed were pruned precociously. At $4 \mathrm{~h} \mathrm{APF} \mathrm{(after}$ pupal formation), before pruning begins in earnest in uninjured arbors, regenerated arbors have already fragmented many branches (Fig. 4E). At $7 \mathrm{~h}$ AFP, when uninjured arbors are beginning to sever primary branches at points proximal to the cell body, many regenerated arbors have already completed the whole process of pruning, including fragmentation of the dendrites and clearance of the debris. Pruning of regenerated dendrites not only is precocious but also occurs in a different sequence than uninjured dendrites. Normally, branch severing proximally to the cell body is the first step in pruning, followed by fragmentation of the distal detached dendrites. However, in regenerated dendrites, fragmentation of the distal dendrites occurs before severing. Thus, one consequence of the alterations seen in regenerated dendrites is defects in later arbor pruning.

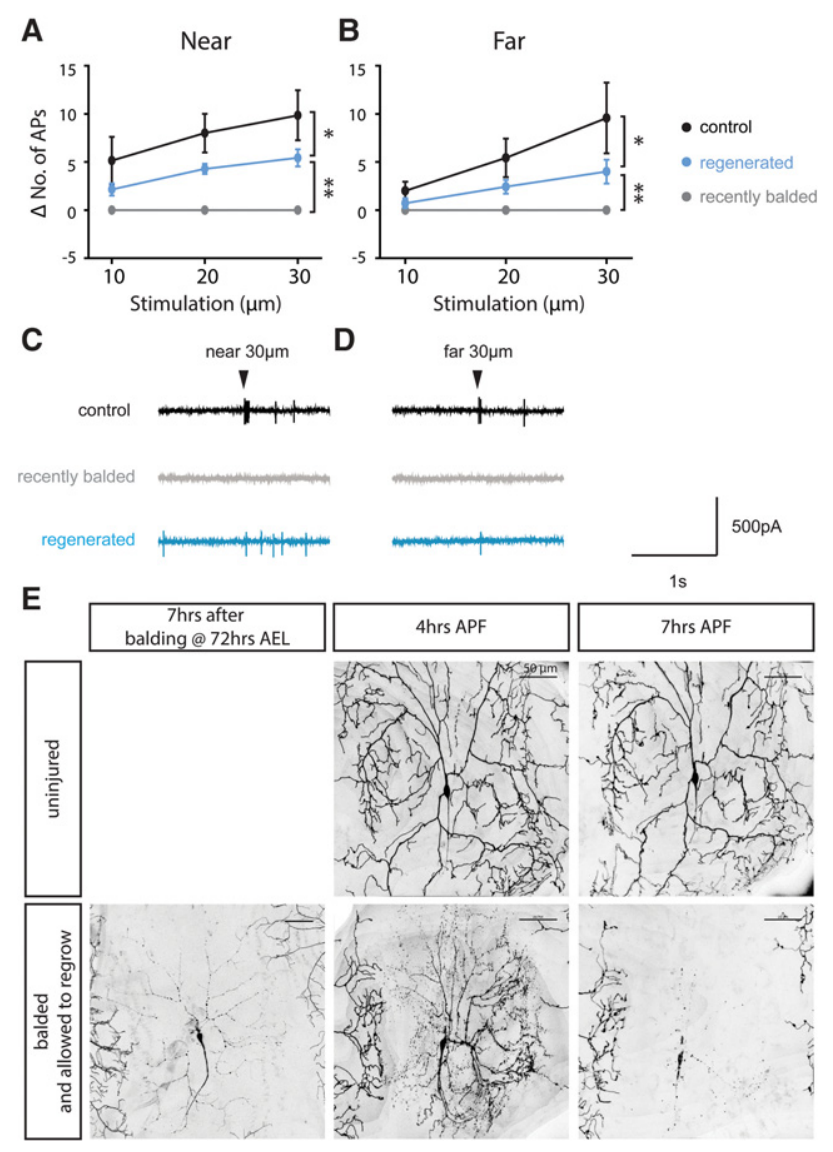

Figure 4. Alterations in regenerated dendrite arbors change receptive function and later pruning. $(A-D)$ Regenerated class III ddaA dendrites (blue) respond to gentle touch stimuli both near $(A)$ and far $(B)$ from the cell body compared with control (black) or recently balded (gray) neurons. $n=7$ control neurons, 7 regenerated neurons, and 7 recently balded neurons. $\left(^{*}\right) P<0.05$; $\left(^{* *}\right)$ $P<0.0001$, by two-way ANOVA. $(C, D)$ Representative traces of recording from class III neurons. Arrowheads indicate the time of $30-\mu \mathrm{m}$ stimulus application either near $(C)$ or far from $(D)$ the cell body. $(E)$ Regenerated class IV dendrites were pruned precociously by fragmentation. (Top) A control uninjured ddaC neuron began pruning by severing the proximal dendrites $\sim 7 \mathrm{~h}$ after pupal formation (APF). (Bottom) A regenerated dendrite arbor was pruned by fragmentation of the distal dendrites and had completed the process by $7 \mathrm{~h}$ APF. Bar, $50 \mu \mathrm{m} . n=15$ balded neurons and 19 mock neurons. 
In light of the altered pruning, we wondered whether those neurons that have already regenerated a dendrite arbor once following injury were capable of regrowing another arbor in the adult. To examine this, we balded larval neurons, allowed them to regenerate in the larvae and then go through pruning during metamorphosis, and subsequently examined the adult neuron. We used the v'ada subtype of class IV da neurons, whose dendrite arbors in the adult are easily imaged on the pleura or underbelly of the abdomen (Shimono et al. 2009). We found that adult neurons that had gone through an extra round of regeneration during the larval stages were indistinguishable from neurons that had never been injured (Supplemental Fig. S4). Thus, dendrite regeneration during the larval phases does not affect the ability of the neuron to regenerate a second time during later metamorphosis.

\section{Electrical activity plays a key role in regeneration}

What is the mechanism that allows neurons to sense that their dendrites have been injured? Uninjured dendrites are depolarized in response to sensory stimuli, but, after dendrite balding, neurons without dendrites will no longer be depolarized by sensory stimuli (as shown in Fig. 4A-D); thus, we hypothesized that electrical activity might play a role in the detection of dendrite injury. In order to test the role of electrical activity in dendrite regeneration, we overexpressed the inward rectifier $\mathrm{K}^{+}$channel Kir2.1, also known as KCNJ2, in the class I and class IV da neurons. Overexpression of this channel electrically silences neurons by hyperpolarizing them-reducing the probability of action potential firing-but does not affect spontaneous neurotransmitter release (Baines et al. 2001). In uninjured class IV da neurons, expression of Kir2.1 caused a reduction in branching, most obvious in older neurons, but did not decrease the total arbor length (Fig. 5A-D). In uninjured class I da neurons, Kir2.1 expression did not change dendrite development at all (Supplemental Fig. S5E-H). Neuronal activity regulates dendrite spines and dendrite maintenance in other systems (Wong and Ghosh 2002), but we found that neuronal activity plays only a minor role in the development of these da neurons.

In comparison with these mild developmental effects on uninjured neurons, Kir2.1 expression drastically reduced regeneration after injury. In class IV da neurons, Kir2.1 expression almost completely blocked dendrite branching and prevented any increase in total dendrite length after injury (Fig. 5A-D). In class I da neurons, Kir2.1 expression markedly reduced the amount of branching after injury (such that these neurons did not regenerate enough branches to catch up to uninjured neurons of the same genotype) and also diminished the lengthening of the dendrite arbor (Fig. 5E; Supplemental Fig. S5E-H). In contrast to the manipulations that equally change both developmental and regenerative branching (Supplemental Fig. S2), Kir2.1-expressing neurons have an underregeneration phenotype without a corresponding developmental underbranching phenotype.

The decreased regenerative growth of Kir2.1-expressing class IV da neurons after injury results in dendrite arbors that cover less body wall territory than either uninjured Kir2.1-expressing neurons or regenerated wild-type neurons (Fig. 5D). We found that other manipulations that electrically inactivate the neuron by altering potassium, such as overexpression of the outward rectifier $\mathrm{K}^{+}$channel ORK or the Shaker $\mathrm{K}^{+}$channel EKO (White et al. 2001; Nitabach et al. 2002), also prevent class IV da neurons from properly regenerating their dendrites after balding injury, resulting in decreased coverage of body wall territory (Fig. 5D; Supplemental Fig. S5A-D). Altogether, this suggests that electrical signaling of da neurons plays a key role in dendrite regeneration after injury.

To test whether subacute inactivation would be sufficient to block regeneration, we used the GeneSwitch system to drive expression of Kir2.1 only after treatment with the drug RU486 (Nicholson et al. 2008), starting 24 h before dendrite balding to allow the neuron to differentiate and develop normally. We verified Kir2.1 expression by imaging the Kir2.1-GFP (Fig. 5F, insets). RU486-induced expression of Kir2.1 caused no significant defects to uninjured neurons but almost entirely blocked dendrite outgrowth after injury, similar to the effect of chronic Kir2.1 expression (Fig. 5F,G), indicating that Kir2.1 expression during and subsequent to the injury is sufficient to block dendrite regeneration.

Dendrite balding does not alter dendritic characteristics or cell fate

Are the neurites that the neurons extend after dendrite balding actually dendrites? Previous reports suggested that regenerated neurites are in fact dendrites based on the cytoskeleton and cytoskeleton-associated proteinsspecifically the orientation of microtubule polarity, localization of the Apc2-GFP transgene, and a requirement for dynein transport (Stone et al. 2014). To further address this question, we found that, in class III neurons, the presence of these neurites is required for firing action potentials in response to a mechanical stimulus (Fig. 4A-D). Moreover, in class IV neurons, we found that the DEG/ ENaC channels ppk1 and ppk26, which are necessary for the function of the dendrites in mechanical nociception (Zhong et al. 2010; Gorczyca et al. 2014; Guo et al. 2014; Mauthner et al. 2014), are properly trafficked into regenerated dendrites and properly excluded from the axon (Fig. $6 \mathrm{~B}, \mathrm{C})$. Thus, our evidence suggests that these neurons are functionally acting as dendrites.

Are alterations in dendrite patterning due to cell fate changes caused by injury? To test for possible changes in cell type specification caused by injury, we immunostained for transcription factors that play a role in class specification and are highly expressed in subsets of da neurons (Grueber et al. 2003a; Dubois et al. 2007; Hattori et al. 2007; Jinushi-Nakao et al. 2007). We found no difference in expression levels of the class IV-specific transcription factors cut or knot/collier $6 \mathrm{~h}$ after balding class IV da neurons (Fig. 6A; Supplemental Fig. S6B). We also looked at abrupt, which is highly expressed in class I da neurons, and found no difference in abrupt expression $72 \mathrm{~h}$ after balding class I da neurons (Supplemental Fig. S6A). 
Thus, the expression of these transcription factors, which determine the different da neuron classes, seems to be unaffected by dendrite balding.

In addition to transcription factors, we tested for balding-induced alterations in cell fate by looking at the expression of sensory receptors that are specific to different subtypes of da neurons. The DEG/ENaC channels ppk1 and ppk26, which are necessary for mechanical nociception, are normally specifically expressed by class IV da neurons (Zhong et al. 2010; Gorczyca et al. 2014; Guo et al. 2014; Mauthner et al. 2014). We showed above that these proteins are properly trafficked into regenerated dendrites

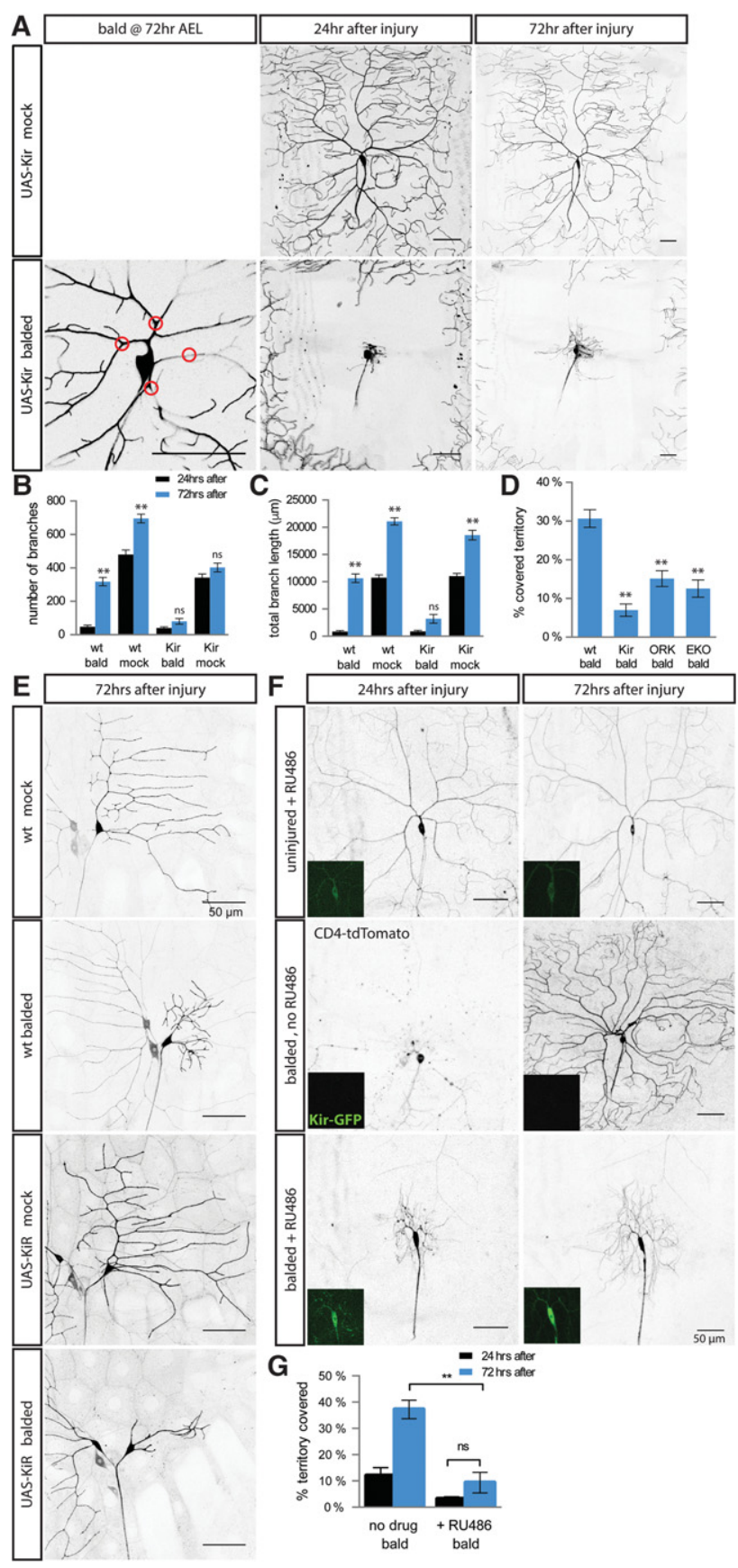

and excluded from axons, but are they still expressed by the correct neuronal subtype? We found that, after balding, these class IV-specific channels are still specifically expressed in the class IV da neurons by immunostaining (Fig. 6B,C). We also tested for the expression of NOMPC, a mechanotransduction channel subunit expressed in class III da neurons but not class IV da neurons (Cheng et al. 2010; Yan et al. 2013). NOMPC was not detectable in either uninjured or regenerated class IV da neurons, while the immunostaining properly marked an adjacent uninjured class III da neuron (Supplemental Fig. S6C).

Together, these data suggest that balding and regeneration do not induce cell type changes in class I or class IV da neurons, but instead the injured neurons retain their cell type identities.

Two-photon dendrite balding minimally affects adjacent glia and epidermal cells

In order to determine how the two-photon injury might affect the environment for regenerating dendrites, we examined the adjacent cell types to see whether they were also injured by the two-photon laser. At the time of injury, at $72 \mathrm{~h} \mathrm{AEL}$, glia wrap the axon and some of the cell body but not the proximal dendrites, so the glia are some distance away from the site of the two-photon injury. The glia have wrapped the axon and the cell body by $24 \mathrm{~h}$ after injury but do not wrap the proximal dendrite branches until $72 \mathrm{~h}$ after injury (Fig. 6D; Han et al. 2011). At $72 \mathrm{~h}$ after

Figure 5. Manipulating electrical activity blocks injury-induced dendrite growth. (A) Class IV neurons expressing Kir2.1 have relatively normal dendrite outgrowth in the absence of injury (top row) but a dramatically reduced growth after balding (bottom row). Red circles indicate points where dendrites will be severed. $(B, C)$ Compared with wild-type neurons and uninjured Kir2.1-expressing neurons, Kir2.1-expressing class IV neurons have dramatically less branch formation $(B)$ and dendrite extension $(C)$ between 24 $\mathrm{h}$ (black) and $72 \mathrm{~h}$ (blue) after balding. $\left.{ }^{* *}\right) P<0.001$ comparing 72 $\mathrm{h}$ with $24 \mathrm{~h}$ after balding or after mock injury by one-way ANOVA with Tukey's multiple comparisons test. $n=14$ balded wild-type neurons, 12 mock wild-type neurons, 17 balded Kir neurons, and 15 mock Kir neurons. $(D)$ Electrically inactivating class IV neurons using $\mathrm{K}^{+}$channel transgenes decreases territory recoverage after

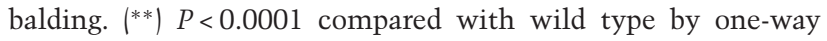
ANOVA with Dunnett's multiple comparisons test. $n=14$ wildtype balded neurons, 17 Kir balded neurons, 20 ORK balded neurons, and 6 EKO balded neurons. (E) Kir expression decreases class I neuron dendrite regeneration after injury without affecting the development of uninjured neurons. $n=24$ wild-type balded neurons, 36 wild-type mock neurons, 20 Kir balded neurons, and 31 Kir mock neurons. $(F, G)$ Defects in injury-induced outgrowth are independent of early dendrite development. (Inset) Twenty-four hours before injury, flies with ppk-CD4-tdTomato ppk-GS, UASKir2.1-GFP were given RU486 or no drug to induce Kir2.1-GFP expression. $(G)$ While drug treatment without injury did not alter dendrite morphology, RU486-induced Kir2.1 significantly decreased territory recoverage after balding compared with balded neurons with no RU486. (ns) $P>0.05$; $\left(^{* *}\right) P<0.0001$ by one-way ANOVA with Tukey's multiple comparisons test. $n=11$ neurons without RU486 and 10 neurons with RU486. Bar, $50 \mu \mathrm{m}$. 
A
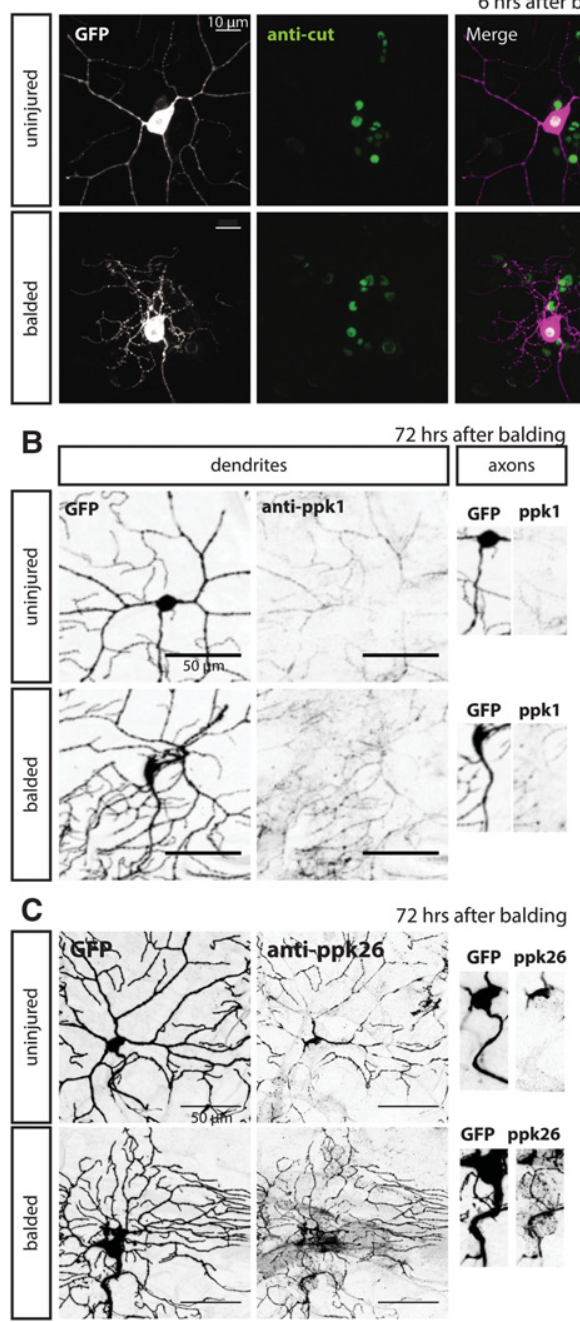

D

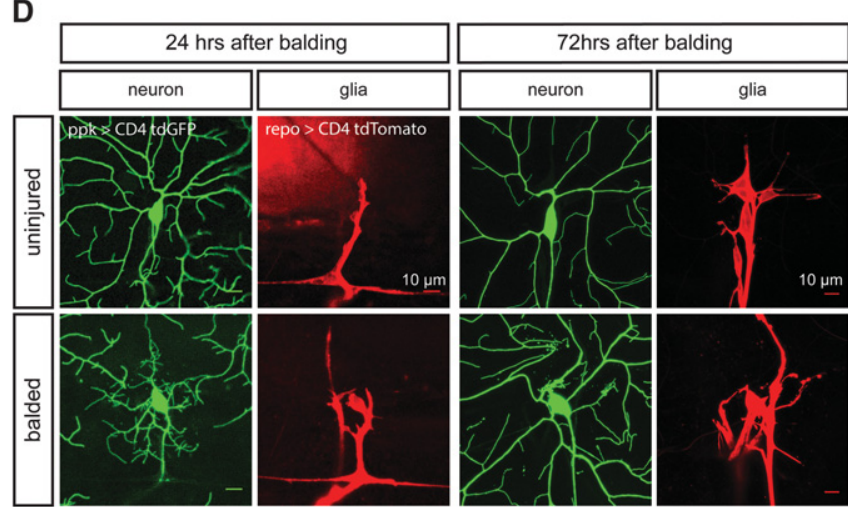

E

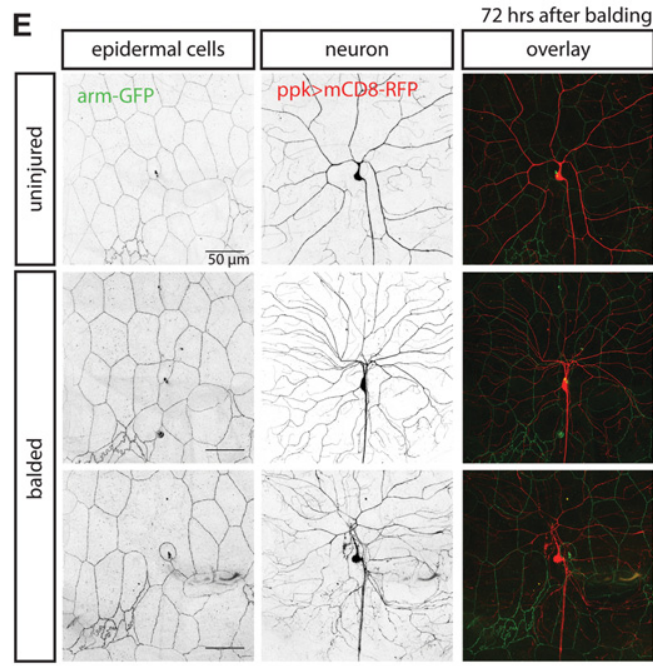

Figure 6. Class IV dendrite balding and regeneration do not permanently alter expression of the class-specific transcription factor cut, receptor sorting and trafficking, glial wrapping, or epidermal cells. $(A)$ Immunostaining for the transcription factor cut did not show significant differences in expression level between neurons $6 \mathrm{~h}$ after balding versus control cells. $(B, C)$ Immunostaining for $p p k 1(B)$ and ppk26 $(C)$ show sorting and trafficking of the mechanoreceptors into the somatodendritic compartment and exclusion from the axon in both uninjured control neurons and neurons $72 \mathrm{~h}$ after balding and regeneration. $(D)$ Glial cells, marked by the repo promoter driving expression of CD4-tdTomato, wrap the axon and cell body at $24 \mathrm{~h}$ after balding and also the proximal dendrites of both control neurons and regenerated neurons at $72 \mathrm{~h}$ after balding. (E) Adjacent epidermal cells whose borders are marked by $\beta$-catenin arm ${ }^{\mathrm{GFP}}$ are sometimes uninjured by balding (middle row) and sometimes ablated by the balding injury (bottom row), but neurons are capable of regenerating dendrites in either case. Bars: $A, D, 10 \mu \mathrm{m} ; B, C, E, 50 \mu \mathrm{m}$.

injury, we saw no gross alterations to their morphology when wrapping uninjured versus regenerated arbors. Thus, changes in glial morphology do not account for alterations in regenerated dendrite morphology.

Epidermal cells form a sheet adjacent to the dendrites and are directly next to the dendrites at the time of injury. The two-photon laser, properly focused on the dendrite branch, should only minimally affect the tissue above and below the branch. Using a $\beta$-catenin armadillo-GFP fusion to mark the outlines of the epidermal cells, we observed that the epidermal cells looked normal in many segments where the neuron had been balded and regenerated (Fig. 6E, middle row). Even in segments where balding had killed the adjacent epidermal cell, the neuron was capable of regenerating its dendrite arbor over that area (Fig. $6 \mathrm{E}$, bottom row). This suggests that the minimal damage that two-photon treatment may cause does not impede growth of dendrites after injury.

\section{Discussion}

\section{A framework for studying dendrite regeneration}

Our work establishes a framework for addressing the central but almost unstudied question of how neurons 
might recover from dendrite injury in intact animals. It had been shown that dendrite regeneration does not require the JNK injury-sensing pathway required for axon regeneration after injury, suggesting substantial differences between axon versus dendrite regeneration (Stone et al. 2014). However, regenerated dendrites have not been examined to see how well they compare with dendrites elaborated by uninjured neurons. We found that PNS neurons have a robust ability to regenerate their dendrites to restore the original number of branches and that somatodendritic sorting and cell fate are unaltered. However, there are a number of essential differences between normal and regenerated dendrites.

The key differences that we identified between uninjured and regenerated dendrites are due to a combination of changes in both the environment and the patterning of dendrite regrowth.

The embryonic environment that young uninjured dendrites encounter during early development is different from the larval environment that regenerating dendrites must later traverse, at the very least in terms of the size of the territory to be covered. During larval development, the size of the territory that each neuron is responsible for increases $>20$-fold, so, over time, more and longer dendrites are required to cover this territory fully. We see that, eventually, neurons balded at later stages are unable to regenerate to meet this increasing demand, as class IV neurons balded at $72 \mathrm{~h}$ AEL cannot form enough branches to recover to uninjured levels. Signaling cues from adjacent cells are also likely to change across developmental time; for example, from the epidermal cells that these dendrites touch (Jiang et al. 2014).

In addition to environmental differences, the intrinsic patterning of dendrite outgrowth is altered after injury. The branches that are formed fail to fully self-avoid, resulting in patterning defects due to deficiencies in both integrin-mediated and DSCAM-mediated self-avoidance. Genetic mutations have been identified that perturb either pathway, but, to the best of our knowledge, this is the first manipulation that affects both self-avoidance pathways. In effect, injury causes these neurons to ignore or override many of the patterning cues that normally constrain their growth in favor of recovering a functional dendritic arbor.

Together, the differences in environment and patterning result in a regenerated arbor architecture that is dramatically different from that of an uninjured neuron. Thus, while all of the PNS neuron subtypes that we tested have the ability to regenerate their dendrites, the regenerated arbors are not likely to function exactly the same as uninjured neurons. This is demonstrated by consequential alterations in functional response to sensory stimuli and precocious pruning during pupation.

\section{Separating dendrite regeneration from dendrite scaling growth}

Because the baseline arbor of these class IV da neurons is dynamically changing during development, in some con- ditions, it is hard to distinguish scaling growth from dendrite regeneration in response to injury. To address this, we (1) performed parallel experiments in class I da neurons whose stable arbors do not form any new branches in the absence of injury, (2) demonstrated that alterations in self-avoidance and pruning are specific to regenerated class IV dendrites, and (3) identified neuronal activity as a pathway that specifically regulates regenerative dendrite growth with only minor effects on normal developmental growth. Thus, only by comparing growth after injury with the growth of uninjured neurons can we determine what is injury-induced growth versus normal dendrite scaling.

\section{Neuronal activity in dendrite maintenance and regeneration}

There is a well-known role for neuronal activity in regulating dendrite spines and dendrite maintenance in other systems (Wong and Ghosh 2002). We found that neuronal activity plays no role in the initial development of class IV da neuron arbors, only a minor role in the later development of class IV da neurons, and seemingly no role in the development or maintenance of class I da neurons. However, hyperpolarizing these neurons by overexpressing $\mathrm{K}^{+}$channels significantly blocks regeneration after injury. Hyperpolarizing these neurons might block their ability to sense an injury signal or respond to an injury signal. Alternatively, hyperpolarizing these neurons might obstruct the function of dendrite maintenance pathways. The idea that dendrite regeneration and maintenance are independent pathways is supported by the evidence that class I neurons overexpressing Kir can maintain their arbors perfectly well but fail to regenerate fully following dendrite injury.

\section{Comparison with other studies of dendrite regeneration}

While research on dendrite regeneration has been extremely limited, it is valuable to compare what we found with how neurons in vivo respond to dendrite injury in other systems. While we observed dendrite regeneration in all of the classes of da neurons that we examined, not all invertebrate neurons are capable of dendrite regeneration. In Caenorhabditis elegans, Chung et al. (2006) used a femtosecond laser to sever the dendrites of the neuron PHA as well as the osmotic avoidance neurons AFD and ASH and did not observe any dendrite regeneration by these neurons or any functional recovery of osmosensation. Wu et al. (2007) also failed to observe any regeneration of severed phasmid neurites of PHB and PHA neurons but did observe dendrite sprouting in half of the cases when the ciliated amphid sensory dendrite of the chemosensory AWB neuron was severed, suggesting that some chemosensory dendrites can regenerate. Therefore, dendrite regeneration is not a guaranteed response to dendrite injury.

In some systems, the distal dendrite branch is not cleared after severing. In leeches, after mechanically or 
optically detaching a neurite branch from the rest of a PD neuron, the proximal neurite grows into the distal fragment's territory even though it is still there (Wang and Macagno 1998). These data suggest that, after branch severing, a defect in self-avoidance mechanisms occurs in either the proximal attached or distal detached neurites. This failure to avoid severed neurites is perhaps comparable with the increase in self-crossing events, both contacting and noncontacting crossings, that we observed in regenerated dendrites of balded class IV neurons. The difference is that, in leeches, self-avoidance fails between an intact proximal neurite and the severed distal neurite, while, in fly larvae, self-avoidance fails between sister dendrite branches of the same fully regenerated dendritic arbor.

It is important to consider that the magnitude of the injury may affect the response of the neuron. When a single dendrite branch of a class IV da neuron was severed, no defect in self-avoidance was observed (Sugimura et al. 2003). Severing a single dendrite branch in vivo in the mouse cortex resulted in no change to the branching architecture of the dendrite proximal to the site of injury, although it may have altered spine morphology (Sacconi et al. 2007a,b).

These studies do not address the question of whether regenerated dendrites retain the ability to form functional synapses. Our work shows that the regenerated dendrites of sensory neurons can recover the ability to respond to mechanical stimuli. As the dendrites of larval da neurons do not form synaptic connections with presynaptic partners, this system cannot be used to address the regeneration of synaptic circuits. Other work suggests that the formation of functional synapses after input loss is possible. For example, recent work in the zebrafish retina suggests that, after postsynaptic neurons are killed, newly born neurons can form synapses with the presynaptic partners but only during a limited time window (Yoshimatsu et al. 2016). More systems to study dendrite regeneration will be required to address whether dendrites regenerated by injured, but not killed, neurons can form functional synapses.

\section{Materials and methods}

See the Supplemental Material for full details on Drosophila strains, two-photon dendrite injury and imaging, quantification of regeneration, immunostaining, analysis of self-crossings, GeneSwitch drug-inducible transgene expression, and electrophysiology used in this study.

\section{Two-photon injury and imaging}

Dendrites were severed from da neurons by focusing a Chameleon two-photon 930-nm laser mounted on a custom-built Zeiss fluorescence microscope at every primary branch point proximal to the cell body in a modified version of a previous assay (Song et al. 2012; Stone et al. 2014). Neurons were imaged $\sim 24 \mathrm{~h}$ after injury (to confirm that all dendrite branches had been severed) and $72 \mathrm{~h}$ after injury (to assess regeneration). Mock-injured control neurons are uninjured neurons from the same larvae.

\section{Quantification of regeneration}

We traced dendrite arbors using the Simple Neurite Tracer plugin in ImageJ to determine the number of dendrite branch tips and the total length of all the dendrite branches. On these traced arbors, we performed Sholl analysis of dendrite branches crossing circles separated by $5 \mu \mathrm{m}$. To determine percent territory coverage, we measured the total area of the hemisegment by locating the segment boundaries and midline and then measured what fraction of that area had dendrite branches from the neuron of interest. Bar graphs are shown \pm standard error of the mean.

\section{Immunostaining}

Larvae were filleted and fixed for $30 \mathrm{~min}$ in $4 \%$ paraformaldehyde at room temperature after the final imaging time point. Nonpermeabilized staining using PBS exclusively was used for ppk1, ppk26, and NOMPC antibodies; PBS with $0.01 \%$ Triton-X was used for all other immunohistochemistry.

\section{Self-crossings}

Contacting versus noncontacting crossings were determined by imaging anesthetized animals with a $63 \times$ objective with fine $Z$ slices, as before (Han et al. 2012). As the total length within the field of view was different for different genotypes and conditions (Supplemental Fig. S3B), the number of self-crossing events was normalized to the total length within the field of view.

\section{Acknowledgments}

We thank Miriam Beyder and Susan Younger for technical assistance; Yuanquan Song for assistance with the dendrite injury assay; Jennifer Ortega, Brian Yang, David Gorczyca, Yang Xiang, and Wei Zhang for assistance with pilot experiments; Jay Parrish for the arm-GFP flies; the Bloomington Drosophila Stock Center for other fly lines; A. Vincent for providing the anti-Collier antibodies; and the members of the Jan laboratory for scientific discussions and advice. The authors were supported by F32NS084616 (K.L.T.-P.), the University of California Office of the President Post-doctoral Fellowship Program (K.L.T.-P.), and R37NS040929 (Y.N.J.). L.Y.J. and Y.N.J. are investigators of the Howard Hughes Medical Institute. K.L.T.-P. and Y.N.J. conceived and designed the study. K.L.T.-P. performed the experiments with L.D. for the immunostaining experiments and with T.L. for the class III neuron electrophysiology. K.L.T.-P. analyzed the data. K.L.T.-P., L.Y.J., and Y.N.J. wrote the paper. All of the authors discussed the results and commented on the manuscript.

\section{References}

Baines RA, Uhler JP, Thompson A, Sweeney ST, Bate M. 2001. Altered electrical properties in Drosophila neurons developing without synaptic transmission. J Neurosci 21: 1523-1531.

Barnes AP, Polleux F. 2009. Establishment of axon-dendrite polarity in developing neurons. Annu Rev Neurosci 32: 347-381.

Brower DL. 2003. Platelets with wings: the maturation of Drosophila integrin biology. Curr Opin Cell Biol 15: 607-613.

Brown CE, Wong C, Murphy TH. 2008. Rapid morphologic plasticity of peri-infarct dendritic spines after focal ischemic stroke. Stroke 39: 1286-1291.

Cheng LE, Song W, Looger LL, Jan LY, Jan Y-N. 2010. The role of the TRP channel NompC in Drosophila larval and adult locomotion. Neuron 67: 373-380. 
Chung SH, Clark DA, Gabel CV, Mazur E, Samuel ADT. 2006. The role of the AFD neuron in C. elegans thermotaxis analyzed using femtosecond laser ablation. BMC Neurosci 7: 30.

Crozatier M, Vincent A. 2008. Control of multidendritic neuron differentiation in Drosophila: the role of Collier. Dev Biol 315: 232-242.

Dubois L, Enriquez J, Daburon V, Crozet F, Lebreton G, Crozatier M, Vincent A. 2007. Collier transcription in a single Drosophila muscle lineage: the combinatorial control of muscle identity. Development 134: 4347-4355.

Gao X, Deng P, Xu ZC, Chen J. 2011. Moderate traumatic brain injury causes acute dendritic and synaptic degeneration in the hippocampal dentate gyrus. PLoS One 6: e24566.

Gorczyca DA, Younger S, Meltzer S, Kim SE, Cheng L, Song W, Lee HY, Jan LY, Jan Y-N. 2014. Identification of Ppk26, a $\mathrm{DEG} / \mathrm{ENaC}$ channel functioning with Ppk1 in a mutually dependent manner to guide locomotion behavior in Drosophila. Cell Rep 9: 1446-1458.

Grueber WB, Jan LY, Jan Y-N. 2002. Tiling of the Drosophila epidermis by multidendritic sensory neurons. Development 129: 2867-2878.

Grueber WB, Jan LY, Jan Y-N. 2003a. Different levels of the homeodomain protein cut regulate distinct dendrite branching patterns of Drosophila multidendritic neurons. Cell 112: 805818.

Grueber WB, Ye B, Moore AW, Jan LY, Jan Y-N. 2003b. Dendrites of distinct classes of Drosophila sensory neurons show different capacities for homotypic repulsion. Curr Biol 13: 618-626.

Guo Y, Wang Y, Wang Q, Wang Z. 2014. The role of PPK26 in Drosophila larval mechanical nociception. Cell Rep 9: 1183-1190.

Han C, Jan LY, Jan Y-N. 2011. Enhancer-driven membrane markers for analysis of nonautonomous mechanisms reveal neuron-glia interactions in Drosophila. Proc Natl Acad Sci 108: 9673-9678.

Han C, Wang D, Soba P, Zhu S, Lin X, Jan LY, Jan Y-N. 2012. Integrins regulate repulsion-mediated dendritic patterning of Drosophila sensory neurons by restricting dendrites in a $2 \mathrm{D}$ space. Neuron 73: 64-78.

Han C, Song Y, Xiao H, Wang D, Franc NC, Jan LY, Jan Y-N. 2014. Epidermal cells are the primary phagocytes in the fragmentation and clearance of degenerating dendrites in Drosophila. Neuron 81: 544-560.

Hattori Y, Sugimura K, Uemura T. 2007. Selective expression of Knot/Collier, a transcriptional regulator of the EBF/Olf-1 family, endows the Drosophila sensory system with neuronal class-specific elaborated dendritic patterns. Genes Cells 12: 1011-1022.

Hori N, Carpenter DO. 1994. Functional and morphological changes induced by transient in vivo ischemia. Exp Neurol 129: 279-289.

Hughes ME, Bortnick R, Tsubouchi A, Bäumer P, Kondo M, Uemura T, Schmucker D. 2007. Homophilic Dscam interactions control complex dendrite morphogenesis. Neuron 54: 417-427.

Jan Y-N, Jan LY. 2010. Branching out: mechanisms of dendritic arborization. Nat Rev Neurosci 11: 316-328.

Jiang N, Soba P, Parker E, Kim CC, Parrish JZ. 2014. The microRNA bantam regulates a developmental transition in epithelial cells that restricts sensory dendrite growth. Development 141: 2657-2668.

Jinushi-Nakao S, Arvind R, Amikura R, Kinameri E, Liu AW, Moore AW. 2007. Knot/Collier and cut control different aspects of dendrite cytoskeleton and synergize to define final arbor shape. Neuron 56: 963-978.
Kanamori T, Kanai MI, Dairyo Y, Yasunaga K-I, Morikawa RK, Emoto K. 2013. Compartmentalized calcium transients trigger dendrite pruning in Drosophila sensory neurons. Science 340: $1475-1478$.

Kim MD, Jan LY, Jan Y-N. 2006. The bHLH-PAS protein Spineless is necessary for the diversification of dendrite morphology of Drosophila dendritic arborization neurons. Genes Dev 20: 2806-2819.

Kim ME, Shrestha BR, Blazeski R, Mason CA, Grueber WB. 2012. Integrins establish dendrite-substrate relationships that promote dendritic self-avoidance and patterning in Drosophila sensory neurons. Neuron 73: 79-91.

Klapstein GJ, Fisher RS, Zanjani H, Cepeda C, Jokel ES, Chesselet MF, Levine MS. 2001. Electrophysiological and morphological changes in striatal spiny neurons in R6/2 Huntington's disease transgenic mice. J Neurophysiol 86: 2667-2677.

Kuo CT, Jan LY, Jan Y-N. 2005. Dendrite-specific remodeling of Drosophila sensory neurons requires matrix metalloproteases, ubiquitin-proteasome, and ecdysone signaling. Proc Nat1 Acad Sci 102: 15230-15235.

Li P, Murphy TH. 2008. Two-photon imaging during prolonged middle cerebral artery occlusion in mice reveals recovery of dendritic structure after reperfusion. I Neurosci 28: 1197011979.

Matthews BJ, Kim ME, Flanagan JJ, Hattori D, Clemens JC, Zipursky SL, Grueber WB. 2007. Dendrite self-avoidance is controlled by Dscam. Cell 129: 593-604.

Mauthner SE, Hwang RY, Lewis AH, Xiao Q, Tsubouchi A, Wang Y, Honjo K, Skene JHP, Grandl J, Tracey WD. 2014. Balboa binds to pickpocket in vivo and is required for mechanical nociception in Drosophila larvae. Curr Biol 24: 2920-2925.

Murphy TH, Corbett D. 2009. Plasticity during stroke recovery: from synapse to behaviour. Nat Rev Neurosci 10: 861-872.

Nicholson L, Singh GK, Osterwalder T, Roman GW, Davis RL, Keshishian H. 2008. Spatial and temporal control of gene expression in Drosophila using the inducible GeneSwitch GAL4 system. I. Screen for larval nervous system drivers. Genetics 178: 215-234.

Nitabach MN, Blau J, Holmes TC. 2002. Electrical silencing of Drosophila pacemaker neurons stops the free-running circadian clock. Cell 109: 485-495.

Parrish JZ, Xu P, Kim CC, Jan LY, Jan Y-N. 2009. The microRNA bantam functions in epithelial cells to regulate scaling growth of dendrite arbors in Drosophila sensory neurons. Neuron 63: 788-802.

Ramon y Cajal S. 1928. Cajal's degeneration and regeneration of the nervous system (translator May RM). Oxford University Press, New York.

Sacconi L, Masi A, Diana G, Buffelli M, Pavone FS. 2007a. In vivo micro-lesion of single dendrite with femtosecond laser pulses. In Biophotonics 2007: optics in life science, Proceedings of SPIE-OSA Biomedical Optics (ed. Popp J, Von Bally G), Vol. 6633, paper 6633_35. Optical Society of America, Washington, DC.

Sacconi L, O'Connor RP, Jasaitis A, Masi A, Buffelli M, Pavone FS. $2007 \mathrm{~b}$. In vivo multiphoton nanosurgery on cortical neurons. I Biomed Opt 12: 050502.

Schuldiner O, Yaron A. 2015. Mechanisms of developmental neurite pruning. Cell Mol Life Sci 72: 101-119.

Shimono K, Fujimoto A, Tsuyama T, Yamamoto-Kochi M, Sato M, Hattori Y, Sugimura K, Usui T, Kimura K-I, Uemura T. 2009. Multidendritic sensory neurons in the adult Drosophila abdomen: origins, dendritic morphology, and segment- and age-dependent programmed cell death. Neural Dev 4: 37. 
Soba P, Zhu S, Emoto K, Younger S, Yang S-J, Yu H-H, Lee T, Jan LY, Jan Y-N. 2007. Drosophila sensory neurons require Dscam for dendritic self-avoidance and proper dendritic field organization. Neuron 54: 403-416.

Song Y, Ori-McKenney KM, Zheng Y, Han C, Jan LY, Jan Y-N. 2012. Regeneration of Drosophila sensory neuron axons and dendrites is regulated by the Akt pathway involving Pten and microRNA bantam. Genes Dev 26: 1612-1625.

Stone MC, Albertson RM, Chen L, Rolls MM. 2014. Dendrite injury triggers DLK-independent regeneration. Cell Rep 6: 247-253.

Sugimura K, Yamamoto M, Niwa R, Satoh D, Goto S, Taniguchi M, Hayashi S, Uemura T. 2003. Distinct developmental modes and lesion-induced reactions of dendrites of two classes of Drosophila sensory neurons. J Neurosci 23: 3752-3760.

Wang H, Macagno ER. 1998. A detached branch stops being recognized as self by other branches of a neuron. I Neurobiol 35: $53-64$.

White BH, Osterwalder TP, Yoon KS, Joiner WJ, Whim MD, Kaczmarek LK, Keshishian H. 2001. Targeted attenuation of electrical activity in Drosophila using a genetically modified K (+) channel. Neuron 31: 699-711.

Williams DW, Truman JW. 2005. Cellular mechanisms of dendrite pruning in Drosophila: insights from in vivo time-lapse of remodeling dendritic arborizing sensory neurons. Development 132: 3631-3642.
Wong ROL, Ghosh A. 2002. Activity-dependent regulation of dendritic growth and patterning. Nat Rev Neurosci 3: 803-812.

Wu Z, Ghosh-Roy A, Yanik MF, Zhang JZ, Jin Y, Chisholm AD. 2007. Caenorhabditis elegans neuronal regeneration is influenced by life stage, ephrin signaling, and synaptic branching. Proc Natl Acad Sci 104: 15132-15137.

Xiong Y, Mahmood A, Chopp M. 2013. Animal models of traumatic brain injury. Nat Rev Neurosci 14: 128-142.

Yan Z, Zhang W, He Y, Gorczyca D, Xiang Y, Cheng LE, Meltzer S, Jan LY, Jan Y-N. 2013. Drosophila NOMPC is a mechanotransduction channel subunit for gentle-touch sensation. $\mathrm{Na}$ ture 493: 221-225.

Yoshimatsu T, D'Orazi FD, Gamlin CR, Suzuki SC, Suli A, Kimelman D, Raible DW, Wong RO. 2016. Presynaptic partner selection during retinal circuit reassembly varies with timing of neuronal regeneration in vivo. Nat Commun 7: 10590.

Zhong L, Hwang RY, Tracey WD. 2010. Pickpocket is a DEG/ $\mathrm{ENaC}$ protein required for mechanical nociception in Drosophila larvae. Curr Biol 20: 429-434.

Zonouzi M, Scafidi J, Li P, McEllin B, Edwards J, Dupree JL, Harvey L, Sun D, Hübner CA, Cull-Candy SG, et al. 2015. GABAergic regulation of cerebellar NG2 cell development is altered in perinatal white matter injury. Nat Neurosci 18: 674-682. 


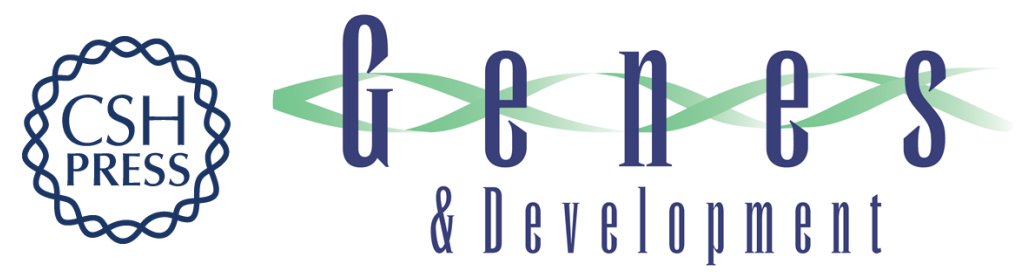

\section{In vivo dendrite regeneration after injury is different from dendrite development}

Katherine L. Thompson-Peer, Laura DeVault, Tun Li, et al.

Genes Dev. 2016, 30:

Access the most recent version at doi:10.1101/gad.282848.116

\section{Supplemental http://genesdev.cshlp.org/content/suppl/2016/08/19/30.15.1776.DC1 \\ Material}

References This article cites 52 articles, 14 of which can be accessed free at:

http://genesdev.cshlp.org/content/30/15/1776.full.html\#ref-list-1

Creative This article is distributed exclusively by Cold Spring Harbor Laboratory Press for the first Commons six months after the full-issue publication date (see

License http://genesdev.cshlp.org/site/misc/terms.xhtml). After six months, it is available under a Creative Commons License (Attribution-NonCommercial 4.0 International), as described at http://creativecommons.org/licenses/by-nc/4.0/.

Email Alerting Receive free email alerts when new articles cite this article - sign up in the box at the top Service right corner of the article or click here.

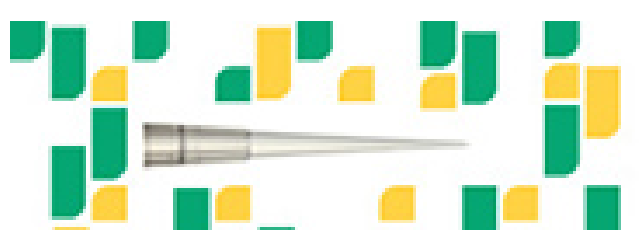

Focused on your science. 